\title{
Analysis of Panax ginseng miRNAs and Their Target Prediction Based on High-Throughput Sequencing
}

Authors

Yingfang Wang ${ }^{1,2}$, Mengyuan Peng ${ }^{1}$, Yanlin Chen ${ }^{1}$, Wenjuan Wang ${ }^{1}$, Zhihua He ${ }^{1}$, Zemin Yang ${ }^{3}$, Zhiyun Lin ${ }^{1}$, Mengjuan Gong ${ }^{1}$, Yongqin Yin ${ }^{1}$, Yu Zeng ${ }^{1}$

Affiliations

1 School of Traditional Chinese Medicine, Guangdong Pharmaceutical University, Guangzhou, Guangdong, China

2 Guangdong Engineering \&Technology Research Center of Topical Precise Drug Delivery System, Guangzhou, Guangdong, China

3 School of Basic Courses, Guangdong Pharmaceutical University, Guangzhou, Guangdong, China

Key words

Panax ginseng, miRNAs, high-throughput sequencing, target gene, cross-kingdom regulation, Araliaceae

received June 19, 2019

revised July 20, 2019

accepted August 3, 2019

Bibliography

DOI https://doi.org/10.1055/a-0989-7302

Published online August 21, 2019 | Planta Med 2019; 85:

1168-1176 @ Georg Thieme Verlag KG Stuttgart · New York | ISSN 0032-0943

Correspondence

Prof. Dr. Yingfang Wang

School of Traditional Chinese Medicine, Guangdong Pharma-

ceutical University

No. 280 Waihuan East Road, Panyu District,

510006 Guangzhou, P. R. China

Phone: + 8602039352174 , Fax: + 8602039352174

150306757@qq.com
Supporting information available online at

http://www.thieme-connect.de/products

\section{ABSTRACT}

Panax ginseng has been widely and effectively used as medicine for thousands of years. However, only limited studies have been conducted to date on ginseng miRNAs. In the present study, we collected 3 ginseng samples from the Changbai Mountain in China. Small RNA libraries were constructed and sequenced on the Illumina HiSeq platform. Sequencing analyses identified 3798 miRNAs, including 298 known miRNAs and 3500 potentially novel miRNAs. The miR166, miR159, and miR396 families were among the most highly expressed miRNAs in all libraries. The results of miRNA expression analyses were validated by qRT-PCR. Target gene prediction through computational and pathway annotation analyses revealed that the primary pathways were related to plant development, including metabolic processes and single-organism processes. It has been reported that plant miRNAs might be one of the hidden bioactive ingredients in medicinal plants. Based on the combined use of RNAhybrid, Miranda, and TargetScan software, a total of 50,992 potential human genes were predicted as the putative targets of 2868 miRNAs. Interestingly, the enriched KEGG pathways were associated with some human diseases, especially cancer, immune system diseases, and neurological disorders, and this could support the clinical use of ginseng. However, the human targets of ginseng miRNAs should be confirmed by further experimental validation. Our results provided valuable insight into ginseng miRNAs and the putative roles of these miRNAs.

\section{Introduction}

Panax ginseng C. A. Meyer, commonly known as Korean ginseng, is a perennial tetraploid plant that belongs to the family Araliaceae. In China, P. ginseng is mostly grown on the Changbai Mountain located in the northeastern part of the country [1,2]. For centuries, $P$. ginseng has been regarded as an important traditional herbal medicine in China because of its high medicinal value. Its medicinal properties include antidiabetic and immunomodulatory effects [3] and enhancement of cognitive performance in Alz- heimer's disease [4]. Ginsenosides, a group of characteristic dammarane triterpene saponins, are the major medicinally relevant compounds found in ginseng [5]. In addition, polysaccharides and phenolics also contribute to the pharmacological effects of ginseng roots. However, the presence of these compounds cannot completely explain the broad clinical effects of ginseng.

miRNAs are a class of small endogenous, single-chain, regulatory RNAs that originate from precursor transcripts with long hairpin structures and are eventually processed to generate small (18-24 nt) miRNA fragments. Recent studies have demonstrated 


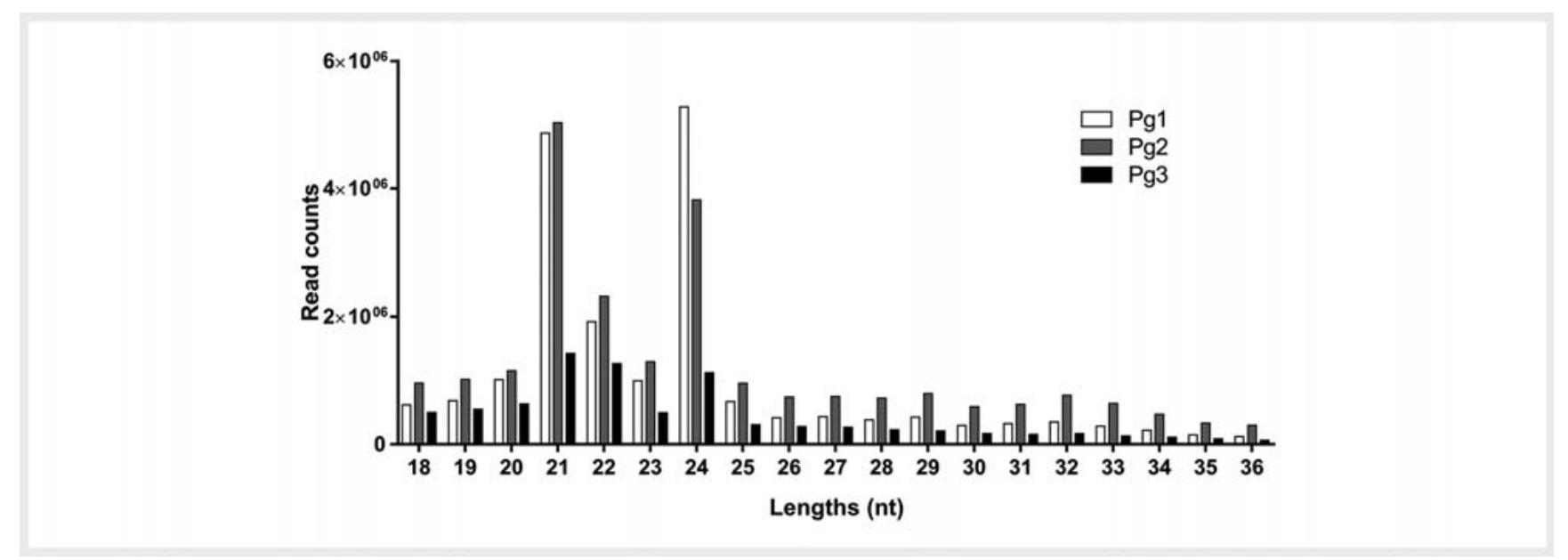

- Fig. 1 Size distribution of small RNAs from 3 ginseng samples detected by high-throughput sequencing.

that miRNAs play key roles in gene expression regulation at the post-transcriptional level through direct cleavage of transcripts, translational repression [6], or, in some cases, methylation at the transcriptional level in plants. Therefore, miRNAs can facilitate the identification of relevant targets for disease diagnosis and treatment.

Modern genetic and genomic studies on medicinal plants are limited. Genomic and transcriptomic data for $P$. ginseng have been recently released [7]. As of June 2017, raw sequencing reads have been deposited in NCBI under the project number PRJNA385956. The increasing evidence supporting miRNA identification and characterization in other plants, such as Arabidopsis thaliana [8] and Panax notoginseng [9], can provide valuable insights into the analysis of miRNAs in $P$. ginseng. However, our understanding of the regulation of ginseng miRNAs remains limited.

Research on the pharmacodynamic mechanisms underlying the medicinal effects of $P$. ginseng has mainly focused on secondary metabolites, such as ginsenosides and polysaccharides, which are the major bioactive compounds present in the plant; however, these compounds cannot fully explain the complex clinical effects of $P$. ginseng. Considering the potential roles of miRNAs as bioactive components that can facilitate the clinical efficacy of $P$. ginseng, we performed small RNA sequencing on $P$. ginseng.

We identified miRNAs and their targets in the roots of $P$. ginseng using bioinformatics-based analysis of small RNA sequencing data. We identified 298 known and 3500 potentially novel miRNAs. Moreover, a total of 2728 miRNAs were annotated with 23,246 targets. Furthermore, the putative human targets were predicted using bioinformatics tools, and their potential roles in human biological pathways and diseases were elucidated. Our results indicate that ginseng miRNAs may exert beneficial effects against some human diseases such as cancer, neurological disorders, and immune system diseases, which could explain the effective medicinal use of $P$. ginseng since ancient times. Additionally, the results provide scientific basis for clinical use of ginseng in modern medicine.

\section{Results}

To study miRNAs in $P$. ginseng sourced from the Changbai Mountain, 3 small RNA libraries named as Pg1, Pg2, and Pg3 were generated. RNAs isolated from the root tissues of 3 ginseng samples (Pg1, Pg2, and Pg3) were analyzed by high-throughput Illumina sequencing. A total of 77,733,908 raw sequence reads were obtained from the 3 small libraries of the ginseng roots using the HiSeq 2500 platform. After removing the adaptors, low-quality sequences, and sequences shorter than $18 \mathrm{nt}, 16,711,989$, 21,479,360, and 6,146,317 clean tags were obtained (Table 1S, Supporting Information) from Pg1, Pg2, and Pg3 libraries, respectively.

The majority of the clean tags had sizes ranging from 20 to $24 \mathrm{nt}$, wherein the majority of the tags had sizes of 21 and $24 \mathrm{nt}$ (- Fig. 1).

All clean tags were aligned against the $P$. ginseng, GeneBank, Rfam, and Repbase databases. RNAs obtained from the 3 samples were classified into different types of RNAs such as ribosomal RNA (rRNA), transfer RNA (tRNA), small nucleolar RNA (snoRNA), and snRNA, and the abundance of each type of RNA was determined. After alignment, a total of $11,859,850,16,105,931$, and 3,266,695 sRNAs in samples Pg1, Pg2, and Pg3, respectively, were annotated (Table 2S, Supporting Information). For $\mathrm{Pg} 1$, $1,093,860$ of the $1,260,127$ unique tags were mapped to the cDNA sequences in the $P$. ginseng genome. For $\mathrm{Pg} 2,973,503$ of the $1,317,331$ unique tags were mapped. For $\mathrm{Pg} 3,432,380$ of the 826,349 unique tags were mapped.

By mapping the unique miRNA mature sequences to miRBase, 2,729, 2,737, and 2,930 unique known miRNA sequences were obtained. Following a manual check, 167, 193, and 198 known miRNAs were identified in $P$. ginseng in $\mathrm{Pg} 1, \mathrm{Pg} 2$, and $\mathrm{Pg} 3$, respectively (Table 2S, Supporting Information).

We identified a total of 298 miRNAs that were categorized under 229 families. By comparing the miRNAs of $P$. ginseng with those from the miRBase database, 73 of the 229 identified miRNA families were categorized into conserved families. Among these conserved families, miR166, miR156, miR159, miR319, and 
a

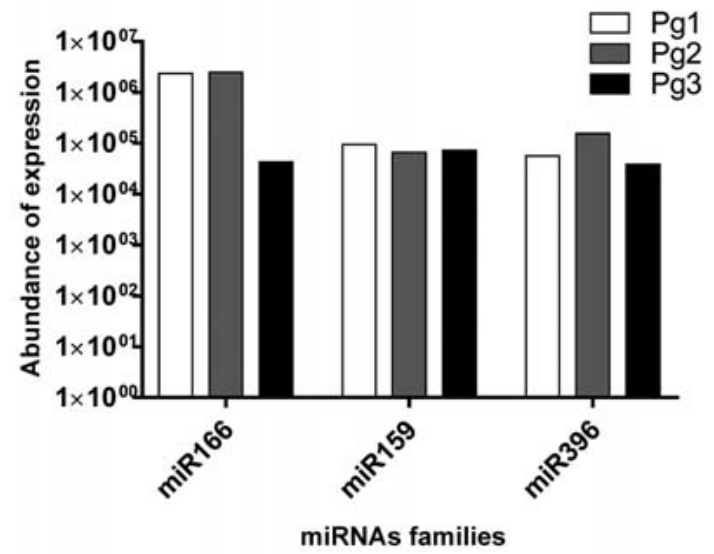

b

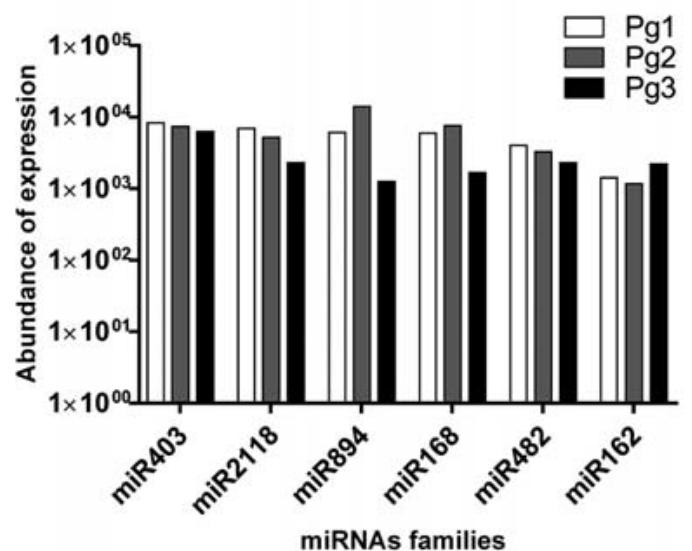

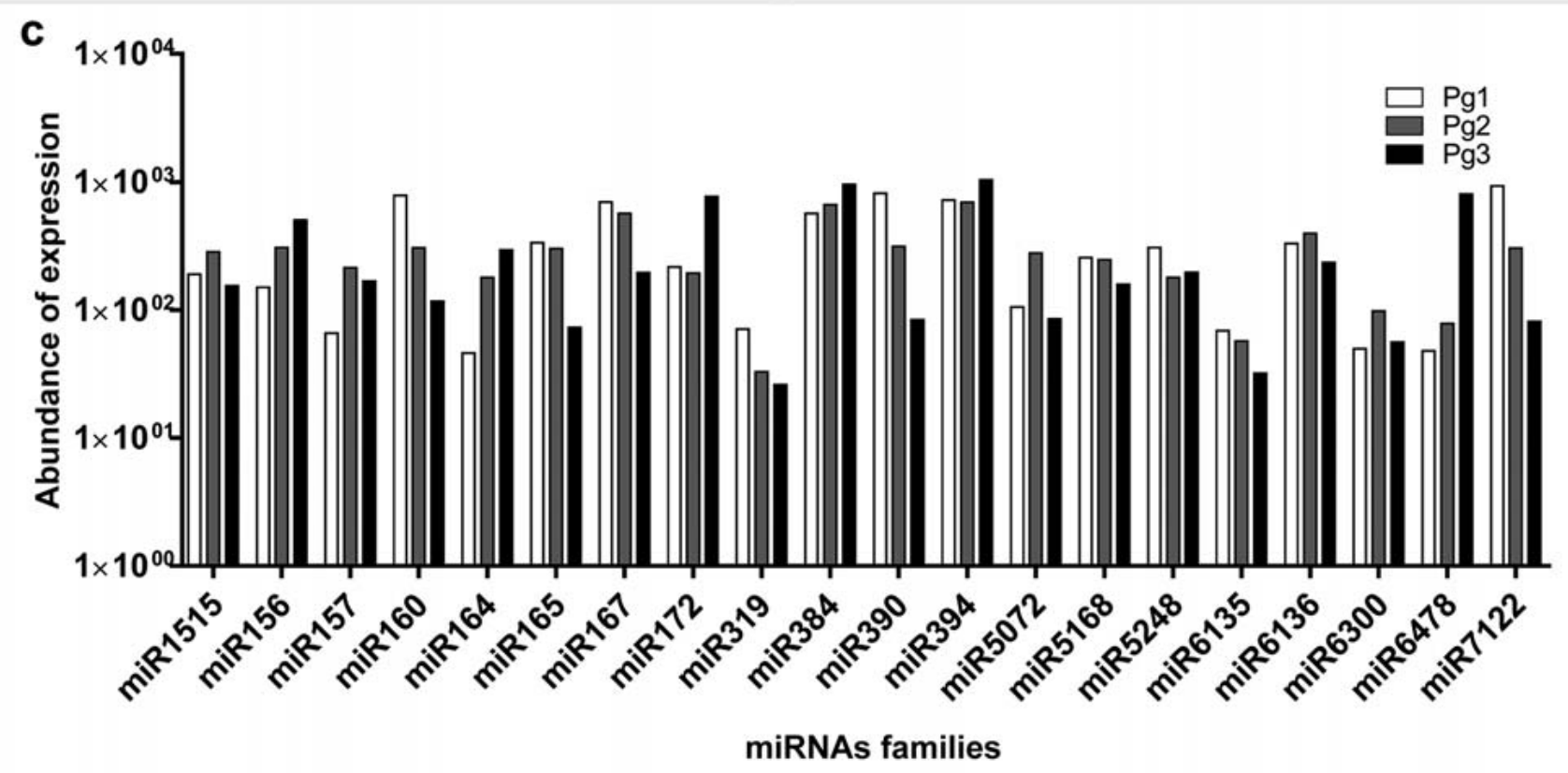

- Fig. 2 Counts of each known miRNA family. a miRNAs with high expression abundances; $\mathbf{b}$ miRNAs with moderate abundances; $\mathbf{c}$ miRNAs with low expression abundances.

miR396, which were highly expressed in more than 4 monocots and dicots, were grouped into highly conserved miRNA families. Contrastingly, miR1515, miR1886, miR165, and miR3630, which were identified in 2 or 3 monocots and dicots were grouped into less conserved miRNAs (Fig. 1S, Supporting Information). In addition, miR156, miR166, miR396, and miR159 from these conserved miRNA families comprised 371, 294, 172, and 114 members, respectively. Moreover, 156 nonconserved miRNA families were detected in P. ginseng, the majority of which consisted of a very small number of family members.

Of the 73 conserved miRNA families, miR396, miR166, and miR159 were the most abundantly expressed miRNAs in the libraries ( $\triangleright$ Fig. 2 a). Several miRNA families, including miR168, miR894, miR403, miR482, miR162, and miR2118, were moder- ately abundant ( $\boldsymbol{F}$ Fig. $\mathbf{2}$ b). Although the miR390, miR156, and miR160 families were highly conserved, they were low in abundance ( $\triangleright$ Fig. 2 c). The lengths of the conserved miRNAs identified varied from 18 to $24 \mathrm{nt}$. Some nonconserved miRNAs such as pgimiR6135, pgi-miR6136, pgi-miR6137, pgi-miR6138, and pgimiR6139 were found only in ginseng species when alignment was made against miRBase v.21 database.

To identify novel miRNA candidates in $P$. ginseng, the unannotated small RNA sequences were matched against the $P$. ginseng genome database using MIREAP_v0.2 software. Based on the secondary structures, DCL1 cleavage sites and minimum free energies of small RNA sequences, 3500 novel miRNAs were predicted and their characteristic hairpin secondary structures were inferred. For Pg1, 2587 novel miRNAs were identified; for Pg2, 
2724 novel miRNAs were identified, and for Pg3, 1411 novel miRNAs were identified. The lengths of these novel miRNAs varied from 18 to $26 \mathrm{nt}$, with the majority being $22 \mathrm{nt}$ long.

Based on the identified known and novel miRNAs, the patmatch_v1.2 program was used to predict the miRNA targets. The analysis identified a total of 23,246 targets out of the 2728 miRNAs in $P$. ginseng.

To elucidate the biological functions of the identified miRNAs, their putative target genes were analyzed based on the Gene Ontology (GO) annotations. All the predicted target genes were categorized into 45 functional groups under the following 3 main categories: cellular component, molecular function, and biological process. Within the biological process category, the most significantly enriched GO terms were metabolic process (GO: 0008152), cellular process (GO: 0009987), and single-organism process (GO: 0044699). Under the cellular component category, the most significantly enriched GO terms were cell (GO: 0005623), cell part (GO: 0044464), and organelle (GO: 0043226). Under the molecular function category, the most significantly enriched GO terms included catalytic activity (GO: 0003824) and binding (GO: 0005488) ( Fig.3a). In addition, Kyoto Encyclopedia of Genes and Genomes (KEGG) pathway enrichment was performed to further understand the functions of the miRNA targets. All target genes were annotated via KEGG databases into 131 different pathways. The bubble chart shows the different KEGG pathway groups with different target numbers, including amino sugar and nucleotide sugar metabolism, starch and sucrose metabolism, and glycerophospholipid metabolism ( Fig. 3 b).

RNAhybrid, Miranda, and TargetScan software was used to predict potential human gene targets for each miRNA. Based on the combined results using the 3 methods, a total of 50,992 potential genes corresponding to 2686 miRNAs were obtained. The proposed targets were used for pathway enrichment analysis, which could provide further insights into the biological functions of the genes. The predicted targets were mapped to the KEGG database and categorized into 296 signaling pathways, of which 24 pathways were significantly enriched ( $\bullet$ Fig. 4 ). Notably, these highly enriched KEGG pathways were found to be associated with some human diseases, especially cancer and neurological disorders.

Three significantly enriched signaling pathways were strongly related to cancer, including bladder cancer (ko05219), small cell lung cancer (ko05222), and central carbon metabolism in cancer (ko05230).

Notably, the putative human genes targeted by ginseng miRNAs were also involved in 2 pathways associated with the nervous system; these pathways included amyotrophic lateral sclerosis (ALS) (ko05014) and phosphatidylinositol signaling system (ko04070). In addition, 4 significantly enriched pathways were found to be related to signal transduction including Hedgehog signaling pathway (ko04340), transforming growth factor beta (TGF- $\beta$ ) signaling pathway (ko04350), Hippo signaling pathway (ko04390), and neuroactive ligand-receptor interaction (ko04080).

In the present study, quantitative reverse transcription polymerase chain reaction ( $q R T-P C R$ ) was employed to validate the re- sults of the high-throughput sequencing because of its high sensitivity, specificity, and rapidity. Specifically, 5 conserved miRNAs were randomly selected for qRT-PCR validation. Results showed that the expression patterns of the selected miRNAs were consistent with those obtained from high-throughput sequencing ( $\bullet$ Fig. 5).

\section{Discussion}

P. ginseng has been clinically used as an effective herbal medicine for several millennia in ancient China. Modern pharmacological research on the active ingredients of $P$. ginseng cannot fully explain its clinical efficacy. miRNAs are a class of noncoding RNAs that play crucial roles in gene expression regulation. miRNAs and their targets in various plant species can be identified by highthroughput sequencing technology. However, there is very limited information available regarding miRNAs and their targets in P. ginseng. In this study, a total of 3798 ginseng miRNAs (298 known and 3500 novel) were identified, and the target genes of the miRNAs were found to be highly enriched terms related to biosynthesis of secondary metabolites, plant-pathogen interactions, and metabolic pathways. The target gene uridine phosphorylase (UDP)-glucosyltransferase (UGT), which accounted for one of the largest gene families of ginseng, is regulated by multiple miRNAs such as miR1140, miR5073, and miR528. The UGT gene (Pq3-O-UGT1) in Asian ginseng and American ginseng encodes a key enzyme involved in the synthesis of ginsenoside Rh2 $[10,11]$. The 62 nd residue of the glycosyltransferase UGT (BL) 1 was identified as a "hot spot" for the 3-OH glycosylation of resveratrol [12], a compound that may have some protective effects against several diseases, such as heart diseases, diabetes, neurological diseases [13], and cancer. The target gene of miR-159 is myb-related transcription factor (MYB). Members of the MYB transcription factor family are known to play roles in plant development and secondary metabolism [14]. MYB families have been reported to regulate the accumulation of saponins in $P$. ginseng [15]. miR164 targets NAC (NAM/ATAF/CUC) transcription factors (NAC), which play a role in the tolerance of dehydration by regulating the genes related to osmotic stress in rose petals [16] and affecting the height of rice by mediating the gibberellic pathway [17].

Recently, researchers have found that plant miRNAs can not only regulate their own gene expression, but also transfer and regulate gene expression in a cross-kingdom manner [18] (i.e., affecting the organism from which they do not originate). In one study, fresh rice, which is abundant in miR168a, was fed to mice, and it was found to be highly enriched in the serum, where it could regulate lipid metabolism in mice [19]. miR2911 from honeysuckle was found to be highly enriched in mice peripheral blood and lungs and targeted influenza viruses to protect the mice from influenza [20]. Plant-derived miR159 significantly suppressed breast cancer cell proliferation by targeting transcription factor 7 (TCF7) [21]. The miRNAs of Cuscuta campestris can target host mRNAs from a different species to regulate their gene expression, suggesting that these miRNAs play a role in the parasitic process of $C$. campestris [22]. Plant derived miR162a specifically inhibits the expression of the Apis mellifera serine/threonine- 


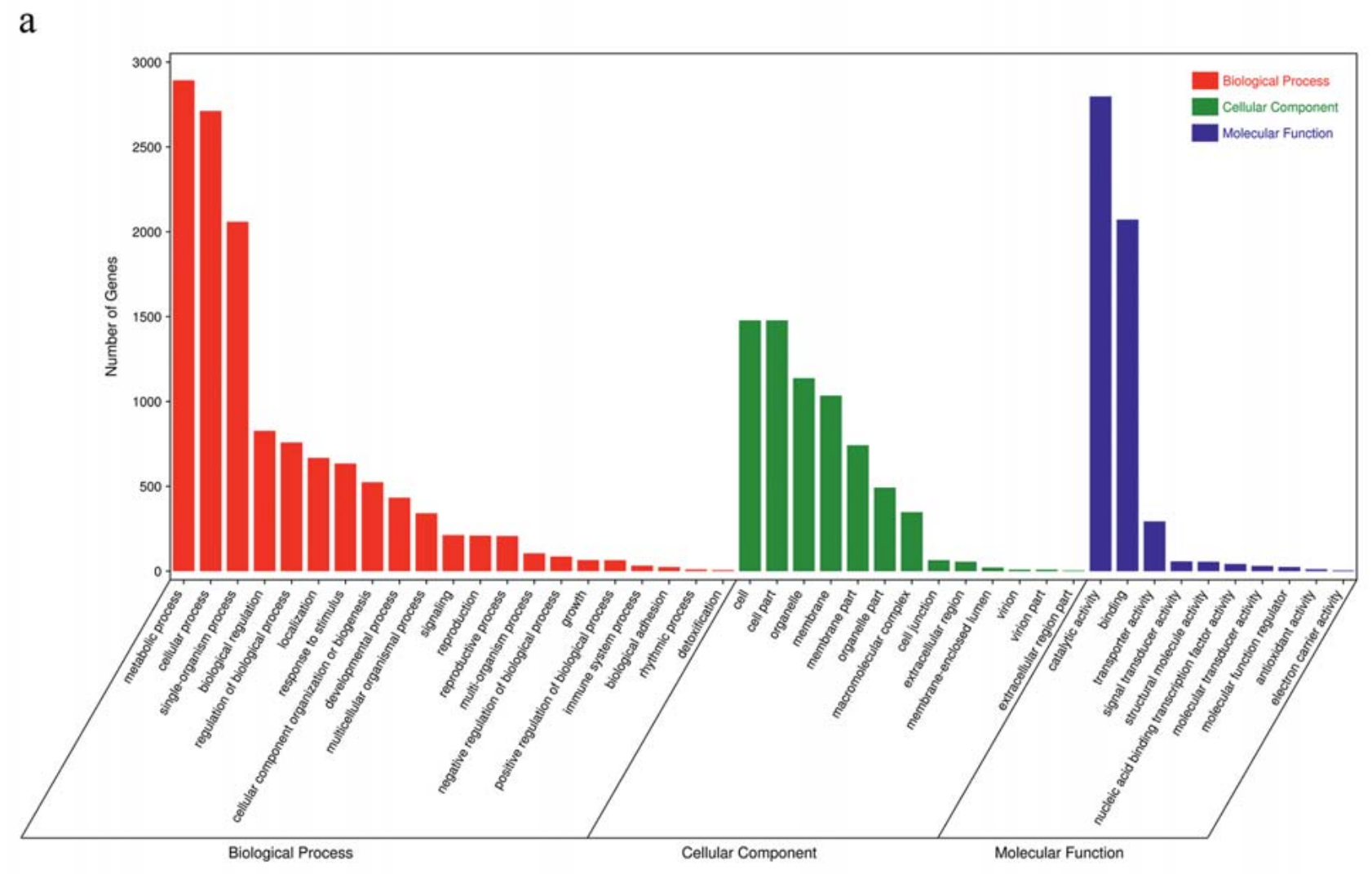

b

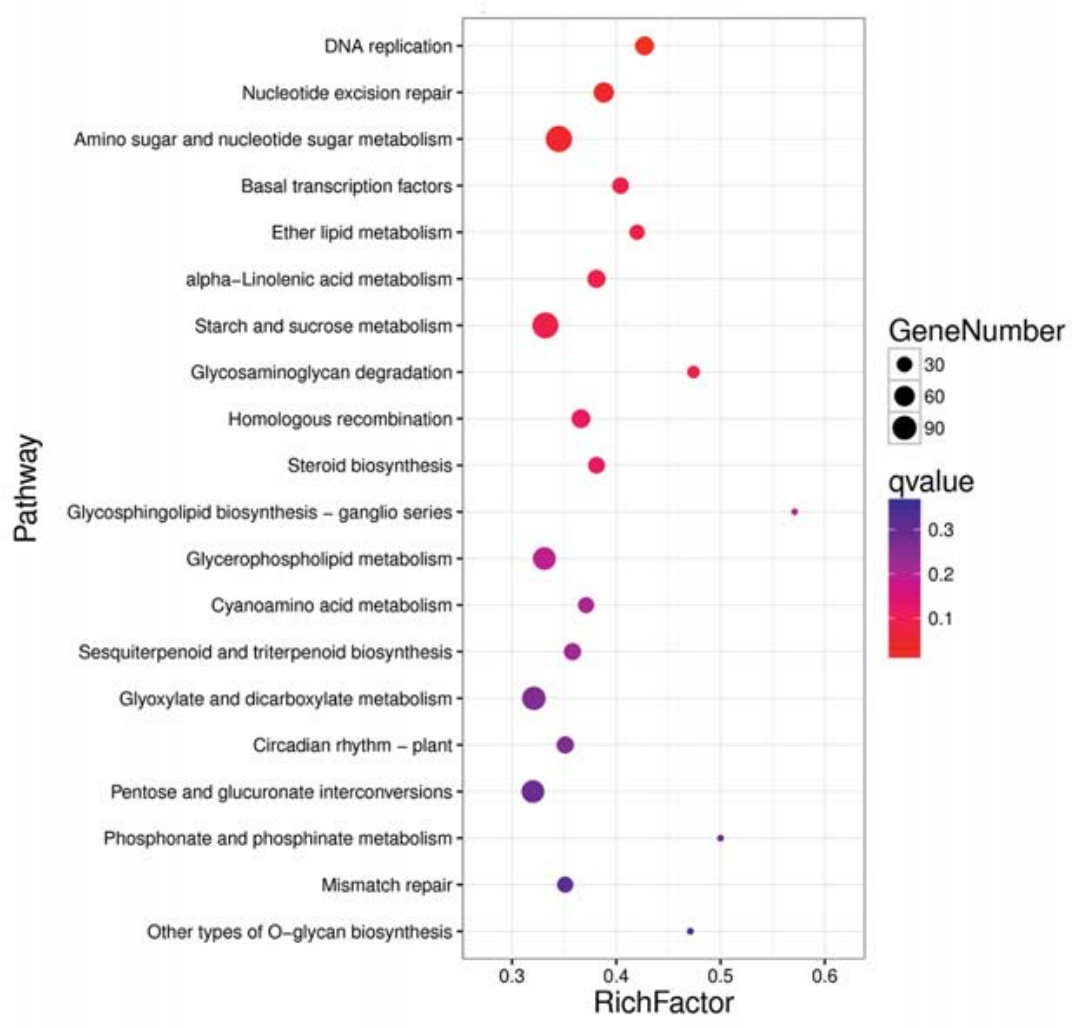

- Fig. 3 GO and KEGG pathway analysis of miRNA targets. a GO enrichment analysis of the miRNA targets; b KEGG enrichment analysis of the miRNA targets. 


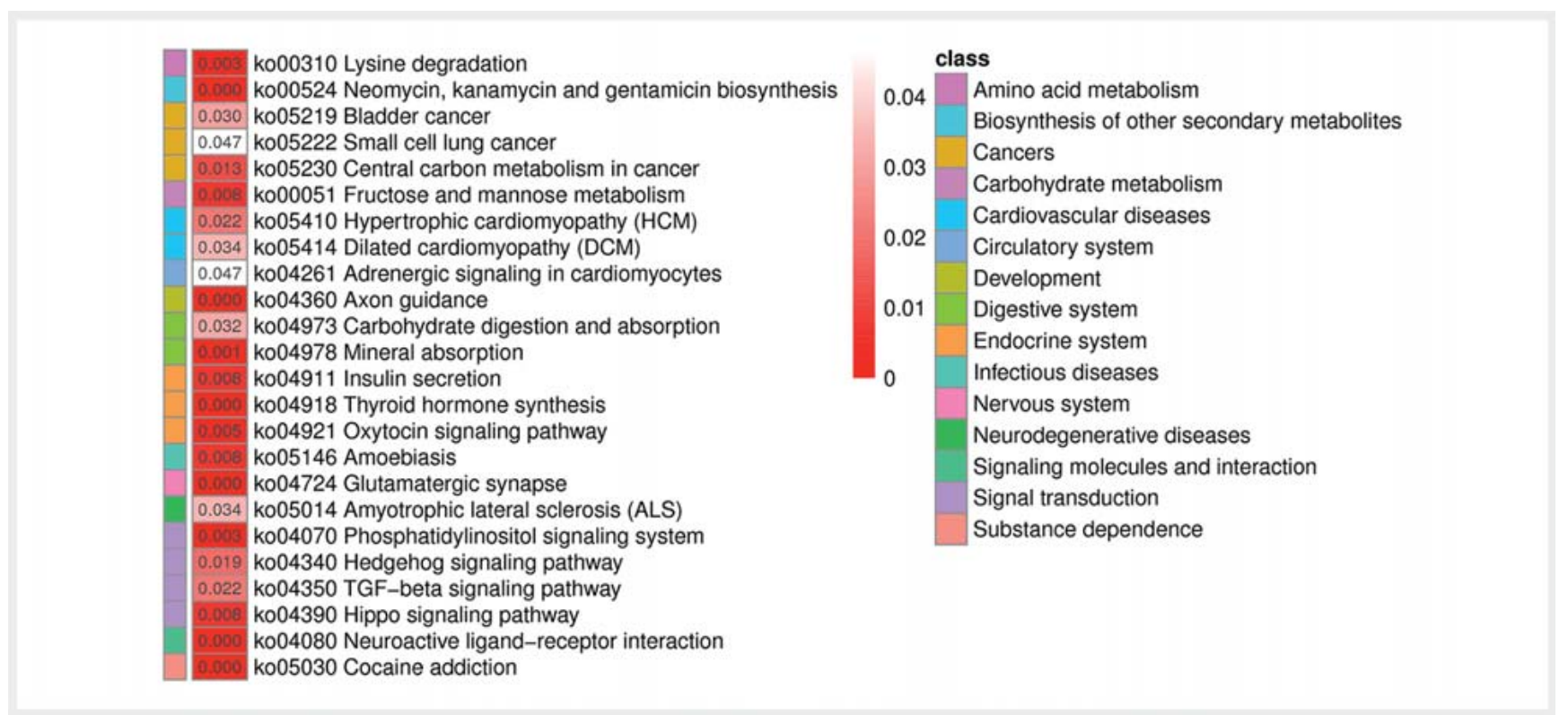

- Fig. 4 Highly enriched KEGG pathways for putative human targets.

protein kinase mTOR (amTOR) gene, which plays an important role in the differentiation of bee larvae [23]. These studies indicated that exogenous plant miRNAs can regulate the expression of target genes in mammals and thus may serve as the basis for further studies on cross-kingdom gene expression regulation by plant miRNAs.

Based on these findings and considering the potential pharmacodynamic roles of ginseng miRNAs, the potential human targets of ginseng miRNAs were identified by bioinformatics prediction. A total of 2686 ginseng miRNAs were assigned to 50,992 putative human target genes, and a total of 296 KEGG pathways were enriched in the gene targets (Table 35, Supporting Information). Among them, 22 pathways were strongly associated with cancer, 24 pathways were associated with immune system diseases, and 15 pathways were related to neurological disorders.

The occurrence and development of tumor involves multiple processes. Aryl hydrocarbon receptor nuclear translocator 2 (ARNT2) is target gene of miR10206 which is known to be involved in carcinogenesis and cancer progression [24]. ARNT 2 is a transcription factor involved in adaptive responses to cellular stress caused by xenobiotic substances [25]. ARNT2 has been reported to regulate tumor growth in oral squamous cell carcinoma and human hepatocellular carcinoma [24] and acts as a potential tumor suppressor gene. The target gene neurotrophic receptor tyrosine kinase 3 (NTRK3) of miR166 is emerging as an important target for cancer therapeutics. NTRK3 can encode the tropomyosin receptor kinase (TRK) family of tyrosine receptor kinases [26]. TRK fusions with other proteins have been implicated in the pathogenesis of many cancer types, including glioblastoma, papillary thyroid carcinoma, and secretory breast carcinomas [27]. Therefore, selective inhibition of TRK signaling could be effective for the treatment of these malignancies.

The target of miR5059 is SH3 domain binding protein 2 (SH3BP2), an adapter protein involved in adaptive and innate im- mune response signaling. Tyrosine phosphorylation of SH3BP2 regulates $B$ cell receptor-mediated activation of nuclear factor of activated T cells [28]. Gain-of-function of SH3BP2 augments inflammation and bone loss by increasing macrophage activation and osteoclast formation [29] while SH3BP2 deficiency inhibits bone loss and the induction of arthritis by disrupting osteoclastogenesis and decreasing autoantibody production [30]. Therefore, modulation of SH3BP2 expression has promising therapeutic potential for the treatment of rheumatoid arthritis. The target gene serpin family A member 1 of miR5059 could encode alpha- 1 antitrypsin, which regulates CD4(+) lymphocyte levels and is rate-limiting in HIV-1 disease [31]. Ras homolog gene family $\mathrm{H}(\mathrm{RhoH})$, the target of miR5072, is a membrane-bound adaptor protein involved in proximal $\mathrm{T}$-cell receptor signaling. $\mathrm{RhoH}$ has been demonstrated to inhibit TH17 differentiation; thus, it plays a role in the pathogenesis of psoriasis [32]. Inducible T cell costimulator (ICOS), the target gene of miR6135, plays a crucial role in various disease-mediated immune responses. ICOS-induced c-Maf was found to regulate IL-21 production, which in turn regulates the expansion of helper T cells and follicular helper T cells [33].

The target gene of miR5272, the sodium voltage-gated channel alpha subunit 1 , which encodes the voltage-gated $\mathrm{Na}+$ channel alpha subunit NaV1.1, is the clinically most relevant epilepsy gene that is associated with several epilepsy syndromes [34], including genetic epilepsy with febrile seizures plus and severe myoclonic epilepsy of infancy. The insulin degrading enzyme (IDE) is the target of miR5073. IDE is a ubiquitous zinc peptidase of the inverzincin family that is capable of degrading several extracellular and intracellular polypeptides, such as $\beta$-amyloid and amylin, thereby contributing to the therapy of neurodegenerative diseases [35], such as Alzheimer's disease.

By targeting essential molecules involved in these pathways, ginseng miRNAs can exert therapeutic effects against cancer, immune system diseases, and neurological disorders. In summary, 


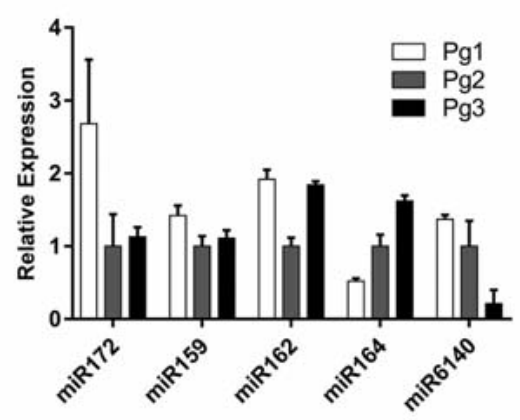

- Fig. 5 The expression levels of 5 randomly selected miRNAs were confirmed by qRT-PCR in $P$. ginseng.

ginseng miRNAs are promising therapeutic agents with clinical efficacy. Our findings provided new insights for the cross-kingdom regulation of ginseng miRNAs. Further studies should be performed to elucidate the role and mechanism underlying the use of $P$. ginseng miRNAs in the treatment of diseases.

Statistics of clean data (Table 1S), categorization of clean tags (Table 2S), KEGG pathways for putative human targets (Table 3S), and distribution of the number of members in conserved miRNA families (Fig. 1S) are available as Supporting Information.

\section{Materials and Methods}

\section{Plant materials and RNA extraction}

Three P. ginseng root samples were collected in September 2015 from the Changbai Mountain in jilin Province in northeast China. The samples included 6- and 15-year-old cultivated ginsengs and a 15 -year-old wild ginseng. They were identified as $P$. ginseng by associate professor Hongyan Ma from Guangdong Pharmaceutical University. The samples are identical to the deposition of $P$. ginseng in Chinese Academy of Sciences Institute of Botany with registration number PE 01471910 . Immediately after collection, the samples were snap-frozen with liquid nitrogen and stored at $-80^{\circ} \mathrm{C}$ for further analysis.

Total RNA was extracted from each sample of $P$. ginseng roots using TRIzol reagent (Invitrogen) according to the manufacturer's instructions. The RNA yield was determined using a NanoDrop 2000 spectrophotometer (Thermo Scientific) and Agilent 2100 BioAnalyzer, and RNA integrity was evaluated by agarose gel electrophoresis and staining with ethidium bromide.

\section{Library construction and sequencing of RNA}

Three libraries were constructed using the extracted RNA according to standard procedures for small RNA library construction and sequencing. Firstly, small RNA was isolated from total RNA by using 15\% PAGE, and 18-30 nt sequence fragments were recovered by gelatinization. In a next step, ligation of the 3 'adaptor and ligation of the 5'adaptor were performed. Then reverse transcription and PCR amplification was carried out to produce CDNA library. Finally, the PCR products were purified by PAGE for subsequent sequencing, and library quality was assessed on the Agilent Bioanalyzer 2100 system. The resulting products were subjected to Illumina HiSeq 2500 sequencing, which was performed at Shanghai OE Biotech Co., Ltd.

\section{Quality control of sequencing data}

Small RNA reads were in the fastq format generated by Illumina (OE Biotech). The basic reads were converted into sequence data (also called raw data) by base calling. Low-quality reads were filtered, and the reads with $5^{\prime}$ primer contaminants and poly $(A)$ were removed using Cutadapt with the error rate (-e) set to 0.1 . From the raw data, reads shorter than $18 \mathrm{nt}$ were filtered out to obtain the clean data. The clean and high-quality data were used for subsequent analyses. The sequence dataset generated in this study is available at the sequence read archive of National Center for Biotechnology Information (NCBI) under the number of PRJNA 553823 .

\section{Identification of known and novel miRNAs}

For primary analysis, the length distribution of small RNAs was determined. The clean tags were aligned with the $P$. ginseng, GeneBank, Rfam, and Repbase database using Bowtie software tools. The noncoding RNAs (ncRNAs), including rRNA, tRNA, snRNA, snoRNA, and other ncRNA, and repeats, were excluded. The known miRNAs were identified by alignment against miRBase v.21 database, and the known miRNA expression patterns in each sample were analyzed. A basic local alignment search tool (BLAST) search was performed against plant mature miRNAs from the miRBase database to identify conserved miRNAs in the generated ginseng small RNA libraries. The frequency of miRNAs in libraries was normalized and expressed as transcripts per million. For the remaining unannotated sRNA sequences, Mireap_v0.2 software was used to predict novel miRNAs by mapping the sequences against the whole $P$. ginseng genome.

\section{Plant target prediction for both known and novel miRNAs}

Targets of all the ginseng miRNAs determined in this study were predicted using the patmatch [36] (v1.2) software with default parameters.

The sequences of predicted target genes were blasted against the main databases $\mathrm{Nr}$ (NCBI nonredundant protein sequences), $\mathrm{Nt}$ (NCBI nonredundant nucleotide sequences), GO, and KEGG. The annotation information of the homologous genes represented the annotation information of the target genes. GO enrichment and KEGG pathway enrichment analyses of target genes were performed using $\mathrm{R}$ based on the hypergeometric distribution.

\section{Human target gene prediction for ginseng miRNAs}

The human mRNA sequences were downloaded from the HGNG dataset (http://www.genenames.org/). Three commonly used animal target prediction algorithms, namely, RNAhybrid (v2.1.2), Miranda (v3.3a), and TargetScan [37] (v7.0), were employed to predict putative human gene targets, and only the genes identified by all 3 software were selected for further analyses. Next, the target genes were mapped to the KEGG database to identify 
significantly enriched metabolic pathways or signal transduction pathways in target genes.

\section{Validation of ginseng miRNAs by qRT-PCR method}

Results of small RNA sequencing were experimentally validated by qRT-PCR. Total RNA was isolated from the roots of $P$. ginseng following the method described above. Gene-specific real time PCR primers for the 5 randomly selected miRNAs were designed using Primer Express 5.0. Total RNA $(0.5 \mu \mathrm{g})$ was reverse-transcribed, and a $10-\mu \mathrm{L}$ reaction volume was used for performing qRT-PCR using the QuantiFast SYBR Green PCR Kit (Qiagen) performed on a LightCycler 480 II real-time PCR Instrument (Roche), based on the manufacturer's protocol. The housekeeping gene U6 was used as a reference, and each qRT-PCR experiment was repeated thrice. The expression levels of miRNAs were normalized to U6 and were calculated using the $2^{-\Delta \Delta \mathrm{Ct}}$ method.

\section{Supporting Information}

Statistics of clean data (Table 1S), categorization of clean tags (Table 2S), KEGG pathways for putative human targets (Table $3 S$ ), and distribution of the number of members in conserved miRNA families (Fig. 1S) are available as Supporting Information.

\section{Acknowledgements}

This work was supported by the National Natural Science Foundation for Young Scholars of China (No. 81403195) and the Natural Science Foundation of Guangdong Province (No. S2013010015418).

\section{Conflict of Interest}

The authors declare that they have no conflict of interest.

\section{References}

[1] Zhao ZF, Wei HY, Guo YL, Gu W. [Potential distribution of Panax ginseng and its predicted responses to climate change]. Ying Yong Sheng Tai Xue Bao 2016; 27: 3607-3615

[2] Li MR, Shi FX, Zhou YX, Li YL, Wang XF, Zhang C, Wang XT, Liu B, Xiao HX, Li LF. Genetic and epigenetic diversities shed light on domestication of cultivated ginseng (Panax ginseng). Mol Plant 2015; 8: 1612-1622

[3] Ru W, Wang D, Xu Y, He X, Sun YE, Qian L, Zhou X, Qin Y. Chemical constituents and bioactivities of Panax ginseng (C.A. Mey.). Drug Discov Ther 2015; 9: 23-32

[4] Lee ST, Chu K, Sim JY, Heo JH, Kim M. Panax ginseng enhances cognitive performance in Alzheimer disease. Alz Dis Assoc Dis 2008; 22: 222-226

[5] Riaz M, Rahman NU, Zia-Ul-Haq M, Jaffar HZE, Manea R. Ginseng: a dietary supplement as immune-modulator in various diseases. Trends In Food Sci Tech 2019; 83: 12-30

[6] Wang J, Mei J, Ren G. Plant microRNAs: biogenesis, homeostasis, and degradation. Front Plant Sci 2019; 10: 360

[7] Xu J, Chu Y, Liao B, Xiao S, Yin Q, Bai R, Su H, Dong L, Li X, Qian J, Zhang J, Zhang Y, Zhang X, Wu M, Zhang J, Li G, Zhang L, Chang Z, Zhang Y, jia Z, Liu Z, Afreh D, Nahurira R, Zhang L, Cheng R, Zhu Y, Zhu G, Rao W, Zhou C, Qiao L, Huang Z, Cheng YC, Chen S. Panax ginseng genome examination for ginsenoside biosynthesis. Gigascience 2017; 6: 1-15

[8] Sirohi G, Khandelwal A, Kapoor M. High-throughput sequencing and differential expression analysis of miRNAs in response to Brassinosteroid treatment in Arabidopsis thaliana. Funct Integr Genomic 2019; 19: $597-$ 615

[9] Zheng Y, Chen K, Xu ZN, Liao PR, Zhang XT, Liu L, Wei KN, Liu DQ, Li YF, Sunkar R, Cui XM. Small RNA profiles from Panax notoginseng roots differing in sizes reveal correlation between miR156 abundances and root biomass levels. Sci Rep-Uk 2017; 7: 9418

[10] Lu C, Zhao SJ, Wang XS. Functional regulation of a UDP-glucosyltransferase gene (Pq3-O-UGT1) by RNA interference and overexpression in Panax quinquefolius. Plant Cell Tiss Org 2017; 129: 445-456

[11] Khorolragchaa A, Kim Y], Rahimi S, Sukweenadhi J, Jang MG, Yang DC. Grouping and characterization of putative glycosyltransferase genes from Panax ginseng Meyer. Gene 2014; 536: 186-192

[12] Fan B, Dong WX, Chen TY, Chu JL, He BF. Switching glycosyltransferase $\mathrm{UGT}(\mathrm{BL}) 1$ regioselectivity toward polydatin synthesis using a semi-rational design. Org Biomol Chem 2018; 16: 2464-2469

[13] Pannu N, Bhatnagar A. Resveratrol: from enhanced biosynthesis and bioavailability to multitargeting chronic diseases. Biomed Pharmacother 2019; 109: 2237-2251

[14] Liu T, Luo T, Guo X, Zou X, Zhou D, Afrin S, Li G, Zhang Y, Zhang R, Luo Z. PgMYB2, a MeJA-responsive transcription factor, positively regulates the dammarenediol synthase gene expression in Panax ginseng. Int J Mol Sci 2019; 20: E2219

[15] Afrin S, Zhu J, Cao H, Huang J, Xiu H, Luo T, Luo Z. Molecular cloning and expression profile of an abiotic stress and hormone responsive MYB transcription factor gene from Panax ginseng. Acta Bioch Bioph Sin 2015; 47: 267-277

[16] Jiang XQ, Zhang CQ, Lu PT, Jiang GM, Liu XW, Dai FW, Gao JP. RhNAC3, a stress- associated NAC transcription factor, has a role in dehydration tolerance through regulating osmotic stress-related genes in rose petals. Plant Biotechnol J 2014; 12: 38-48

[17] Chen X, Lu SC, Wang YF, Zhang X, Lv B, Luo LQ, Xi DD, Shen JB, Ma H, Ming F. OsNAC2 encoding a NAC transcription factor that affects plant height through mediating the gibberellic acid pathway in rice. Plant J 2015; 82: 302-314

[18] Xie WY, Weng A, Melzig MF. MicroRNAs as new bioactive components in medicinal plants. Planta Med 2016; 82: 1153-1162

[19] Zhang L, Hou DX, Chen X, Li DH, Zhu LY, Zhang Y], Li J, Bian Z, Liang XY, Cai X, Yin Y, Wang C, Zhang TF, Zhu DH, Zhang DM, Xu J, Chen Q, Ba Y, Liu J, Wang Q, Chen JQ, Wang J, Wang M, Zhang QP, Zhang JF, Zen K, Zhang CY. Exogenous plant MIR168a specifically targets mammalian LDLRAP1: evidence of cross-kingdom regulation by microRNA ( $\mathrm{vol} 22$, pg 273, 2012). Cell Res 2012; 22: 273-274

[20] Zhou Z, Li XH, Liu JX, Dong L, Chen Q, Liu JL, Kong HH, Zhang QY, Qi X, Hou DX, Zhang L, Zhang GQ, Liu YC, Zhang YJ, Li J, Wang J, Chen X, Wang H, Zhang JF, Chen HL, Zen K, Zhang CY. Honeysuckle-encoded atypical microRNA2911 directly targets influenza A viruses. Cell Res 2015; 25: 39-49

[21] Chin AR, Fong MY, Somlo G, Wu J, Swiderski P, Wu XW, Wang SE. Crosskingdom inhibition of breast cancer growth by plant miR159. Cell Res 2016; 26: 217-228

[22] Shahid S, Kim G, Johnson NR, Wafula E, Wang F, Coruh C, Bernal-Galeano $\mathrm{V}$, Phifer T, dePamphilis CW, Westwood JH, Axtell MJ. MicroRNAs from the parasitic plant Cuscuta campestris target host messenger RNAs. Nature 2018; 553: 82-85

[23] Zhu K, Liu M, Fu Z, Zhou Z, Kong Y, Liang H, Lin Z, Luo ], Zheng H, Wan P, Zhang J, Zen K, Chen J, Hu F, Zhang CY, Ren J, Chen X. Plant microRNAs in larval food regulate honeybee caste development. PLoS Genet 2017; 13: e1006946

[24] Li W, Liang Y, Yang B, Sun H, Wu W. Downregulation of ARNT2 promotes tumor growth and predicts poor prognosis in human hepatocellular carcinoma. J Gastroen Hepatol 2015; 30: 1085-1093

[25] Kimura Y, Kasamatsu A, Nakashima D, Yamatoji M, Minakawa Y, Koike K, Fushimi K, Higo M, Endo-Sakamoto Y, Shiiba M, Tanzawa H, Uzawa K. 
ARNT2 regulates tumoral growth in oral squamous cell carcinoma. J Cancer 2016; 7: 702-710

[26] Khotskaya YB, Holla VR, Farago AF, Mills Shaw KR, Meric-Bernstam F, Hong DS. Targeting TRK family proteins in cancer. Pharmacol Therapeut 2017; 173: 58-66

[27] Lange AM, Lo HW. Inhibiting TRK proteins in clinical cancer therapy. Cancers (Basel) 2018; 10: E105

[28] Shukla U, Hatani T, Nakashima K, Ogi K, Sada K. Tyrosine phosphorylation of 3BP2 regulates $B$ cell receptor-mediated activation of NFAT. J Biol Chem 2009; 284: 33719-33728

[29] Mukai T, Gallant R, Ishida S, Yoshitaka T, Kittaka M, Nishida K, Fox DA, Morita Y, Ueki Y. SH3BP2 gain-of-function mutation exacerbates inflammation and bone loss in a murine collagen-induced arthritis model. PLoS One 2014; 9: e105518

[30] Mukai T, Gallant R, Ishida S, Kittaka M, Yoshitaka T, Fox DA, Morita Y, Nishida K, Rottapel R, Ueki Y. Loss of SH3 domain-binding protein 2 function suppresses bone destruction in tumor necrosis factor-driven and collagen-induced arthritis in mice. Arthritis Rheumatol 2015; 67: 656667

[31] Bristow CL, Babayeva MA, LaBrunda M, Mullen MP, Winston R. alpha(1) Proteinase inhibitor regulates CD4(+) lymphocyte levels and is rate limiting in HIV-1 disease. PLoS One 2012; 7: e31383

[32] Tamehiro N, Nishida K, Sugita Y, Hayakawa K, Oda H, Nitta T, Nakano M, Nishioka A, Yanobu-Takanashi R, Goto M, Okamura T, Adachi R, Kondo K,
Morita A, Suzuki H. Ras homolog gene family H (RhoH) deficiency induces psoriasis-like chronic dermatitis by promoting TH17 cell polarization. J Allergy Clin Immunol 2019; 143: 1878-1891

[33] Bauquet AT, Jin H, Paterson AM, Mitsdoerffer M, Ho IC, Sharpe AH, Kuchroo VK. The costimulatory molecule ICOS regulates the expression of C-Maf and IL-21 in the development of follicular T helper cells and TH-17 cells. Nat Immunol 2009; 10: 167-175

[34] Lal D, Reinthaler EM, Dejanovic B, May P, Thiele H, Lehesjoki AE, Schwarz G, Riesch E, Ikram MA, van Duijn CM, Uitterlinden AG, Hofman A, Steinbock H, Gruber-Sedlmayr U, Neophytou B, Zara F, Hahn A, Genetic Commission of the Italian League against Epilepsy; EuroEPINOMICS CoGIE Consortium, Gormley P, Becker F, Weber YG, Cilio MR, Kunz WS, Krause R, Zimprich F, Lemke JR, Nurnberg P, Sander T, Lerche H, Neubauer BA. Evaluation of presumably disease causing SCN1A variants in a cohort of common epilepsy syndromes. PLoS One 2016; 11: e0150426

[35] Tundo GR, Sbardella D, Ciaccio C, Grasso G, Gioia M, Coletta A, Polticelli F, Di Pierro D, Milardi D, Van Endert P, Marini S, Coletta M. Multiple functions of insulin-degrading enzyme: a metabolic crosslight? Crit Rev Biochem Mol 2017; 52: 554-582

[36] Yan T, Yoo D, Berardini TZ, Mueller LA, Weems DC, Weng S, Cherry JM, Rhee SY. PatMatch: a program for finding patterns in peptide and nucleotide sequences. Nucleic Acids Res 2005; 33: W262-W266

[37] Lewis BP, Shih IH, Jones-Rhoades MW, Bartel DP, Burge CB. Prediction of mammalian microRNA targets. Cell 2003; 115: 787-798 